\title{
Assessment of Gradient-based Point-to-Point ILC for MIMO Systems with Varying Interaction
}

\author{
Thanh V Dinh, Chris T Freeman, Paul Lewin and Ying Tan
}

\begin{abstract}
This paper examines the performance of a gradient-based point-to-point iterative learning control (ILC) algorithm applied to multivariable input, multivariable output (MIMO) systems. Whilst ILC is concerned with tracking a reference trajectory defined over a finite time duration, the point-to-point formulation addresses application domains where the output is not critical at all points over the task duration. The algorithm therefore enforces tracking of only an arbitrary subset of points, with the advantage that the convergence rate increases and input energy decreases as points are removed from the reference. Experimental results presented using a MIMO test facility which can be configured with variable levels of input-output interaction and exogenous disturbance/noise injection confirm the theoretical findings.
\end{abstract}

\section{INTRODUCTION}

Iterative Learning Control (ILC) is a methodology applicable to systems which repeatedly track a reference, $\mathbf{y}_{d}(t)$, defined over a finite interval $0 \leq t \leq T$. The aim is to use past experience to sequentially improve tracking performance over repeated trials of the task. It has been an area of intense research interest in both theoretical and application domains, see, for example, [1] for a recent literature review. However, rather than follow a motion profile defined at all points, in many applications the system output is only critical at a finite set of prescribed time instants. Examples include production line automation, crane control, satellite positioning, and robotic 'pick and place' tasks in which the critical points correspond to the location of the payloads.

The standard ILC framework is able to tackle the point-topoint problem simply by employing an arbitrary reference, $\mathbf{y}_{d}(t)$, which passes through the desired points. However superior results follow if this is coupled with strategies such as Input Shaping in order to suppress vibrations that occur between the critical points. This approach is taken in [2] for a high-acceleration positioning table. An alternative is to use a simpler feedback controller to track $\mathbf{y}_{d}(t)$ and to employ ILC to update parameters within the input shaping filter applied to the reference, as proposed by [3] for control of an industrial robot. Another approach is to develop ILC algorithms which have two separate components; one which ensures tracking of $\mathbf{y}_{d}(t)$, and another which reduces the amplitude of residual vibrations occurring after the point-topoint location is reached [4].

The drawback to all these methods is that they fail to utilize the extra freedom available in ILC design to satisfy

T. Dinh, C. Freeman and Paul Lewin are with the School of Electronics and Computer Science, University of Southampton, Southampton, SO17 1BJ, UK . Email: tdv205@ecs.soton.ac.uk. Y. Tan is with the Electrical and Electronic Engineering Department, University of Melbourne, Parkville, VIC 3010, Australia. additional performance demands. Furthermore, if $\mathbf{y}_{d}(t)$ is designed a priori to meet such performance objectives, these will not be met in practice due to the presence of model uncertainty and noise.

Other approaches to point-to-point motion control have broken away from the standard ILC framework of tracking a static reference defined over $0 \leq t \leq T$, but have only considered the case where a specified position must be reached at time $t=T$, as in, for example, [5], [6], [7], [8], or the case of a movement between two equilibrium points [9]. Whilst these approaches dispense with tracking unnecessary output points, they do not use the resulting freedom to tackle practical performance objectives. A further limitation is that they only consider a single point-to-point movement, rather than a sequence of actions needed to build up complex movements, such as is required in robotic automation and production line assembly.

In this paper an algorithm is derived which enforces tracking of an arbitrary number of point-to-point movements. Analysis shows that this algorithm robustly converges to the minimal input energy solution, and moreover, that this solution reduces as the number of points is removed from the tracking task. At the same time the convergence rate of the algorithm increases as additional points are removed.

\section{PROBLEM FORMULATION}

For any vector $\mathbf{x} \in \mathbb{R}^{n},\|\mathbf{x}\|_{2}=\sqrt{\mathbf{x}^{T} \mathbf{x}}$. For any matrix $A \in \mathbb{R}^{n \times n},\|A\|$ is the induced norm of the vector norm, $\lambda_{i}(A)$ denotes the $i^{t h}$ eigenvalue of $A$, and $\rho(A)=$ $\max _{i}\left|\lambda_{i}(A)\right|$ is the spectral radius of $A$. The $n \times n$ identity and zero matrices are denoted by $I_{n}$ and $0_{n}$ respectively.

Consider the following linear time-invariant (LTI) system

$$
\begin{array}{rlr}
\mathbf{x}(t+1) & =A \mathbf{x}(t)+B \mathbf{u}(t) & \\
\mathbf{y}(t) & =C \mathbf{x}(t)+D \mathbf{u}(t) & \mathbf{x}(0)=\mathbf{x}_{0}
\end{array}
$$

defined over the finite time interval $t \in[0,1,2, \ldots, N-1]$. Here $\mathbf{x}(\cdot) \in \mathbb{R}^{n}, \mathbf{u}(\cdot) \in \mathbb{R}^{m}, \mathbf{y}(\cdot) \in \mathbb{R}^{p}$ are the state, input and output vectors respectively, and the input and output sequences are given by

$$
\begin{aligned}
\mathbf{u}=\left[\mathbf{u}(0)^{T}, \mathbf{u}(1)^{T}, \ldots, \mathbf{u}(N-1)^{T}\right]^{T} & \in \mathbb{R}^{m N} \\
\mathbf{y}=\left[\mathbf{y}(0)^{T}, \mathbf{y}(1)^{T}, \ldots, \mathbf{y}(N-1)^{T}\right]^{T} & \in \mathbb{R}^{p N}
\end{aligned}
$$

The standard ILC framework constructs a series of inputs which drives the system to track a reference sequence

$$
\mathbf{y}_{d}=\left[\mathbf{y}_{d}(0)^{T}, \mathbf{y}_{d}(1)^{T}, \ldots, \mathbf{y}_{d}(N-1)^{T}\right]^{T} \quad \in \mathbb{R}^{p N}
$$


Let $\mathbf{u}_{k}$ and $\mathbf{y}_{k}$ be the input and output vectors respectively on the $k^{t h}$ trial, with $\mathbf{e}_{k}=\mathbf{y}_{d}-\mathbf{y}_{k}$ the tracking error. Then it is necessary to find a sequence of control inputs that satisfies

$$
\lim _{k \rightarrow \infty}\left\|\mathbf{e}_{k}\right\|=0, \quad \lim _{k \rightarrow \infty}\left\|\mathbf{u}_{k}-\mathbf{u}_{d}\right\|=0
$$

where $\mathbf{u}_{d}$ is the unknown desired input sequence corresponding to $\mathbf{y}_{d}$. Over the $k^{t h}$ trial the input/output time-series relationship can be expressed by $\mathbf{y}_{k}=G \mathbf{u}_{k}+\mathbf{y}_{0}$ where

$$
G=\left[\begin{array}{ccccc}
D & 0 & 0 & \cdots & 0 \\
C B & D & 0 & \cdots & 0 \\
C A B & C B & D & \cdots & 0 \\
\vdots & \vdots & \vdots & \ddots & \vdots \\
C A^{N-2} B & C A^{N-3} B & C A^{N-4} B & \cdots & D
\end{array}\right] \in \mathbb{R}^{p N \times m N}
$$

Here $\mathbf{y}_{0}$ is the response to initial conditions whose effect can be absorbed into the reference trajectory, so that without loss of generality it is assumed $\mathbf{y}_{0}=\mathbf{0}$, or equivalently $\mathbf{x}_{0}=\mathbf{0}$.

An ILC update of the form

$$
\mathbf{u}_{k+1}=\mathbf{u}_{k}+L \mathbf{e}_{k}
$$

can be considered as an iterative numerical method to solve the tracking problem, and has been the focus of significant research effort. The update (4) is convergent to a solution if and only if

$$
\rho\left(I_{p N}-G L\right)<1 .
$$

The convergence rate is given by $\frac{1}{\rho(\cdot)}$ and is maximal when $\rho(\cdot)=0$.

\section{A. Point-to-point ILC formulation}

Now consider the point-to-point problem in which the component of the reference $\mathbf{y}_{d}$ corresponding to the $j^{\text {th }}$ plant output is only required to be tracked at a fixed number, $M_{j} \leq N$, of sample instants given by $0 \leq n_{j, 1}<$ $n_{j, 2}<\cdots<n_{j, M_{j}}<N$, which are contained in the set $\mathcal{S}_{j}=\left\{n_{j, 1}, \ldots, n_{j, M_{j}}\right\}$. The tracked points are extracted from $\mathbf{y}_{d}$ and placed in the same order in a point-to-point reference $\mathbf{y}_{r}$ through the operation $\mathbf{y}_{r}=\Phi \mathbf{y}_{d}$. Here $\Phi \in$ $\mathbb{R}^{M \times p N}$ is defined as follows: Introduce $\psi \in \mathbb{R}^{p N}$ with elements

$$
\psi_{i}= \begin{cases}1 & \text { if }\lfloor(i-1) / p\rfloor \in \mathcal{S}_{i-\lfloor(i-1) / p\rfloor p}, \\ 0 & \text { otherwise }\end{cases}
$$

where $\lfloor\cdot\rfloor$ denotes the 'floor' function. This is a vector whose $(i \times p+j)^{t h}$ point is 1 if the $j^{t h}$ output at time $i$ is required to be tracked, and 0 otherwise. $\Phi$ is produced by splitting each non-zero element of $\boldsymbol{\psi}$ into a separate row, giving

$$
\Phi_{i, j}= \begin{cases}1 & \text { if } \psi_{j}=1, \sum_{q=1}^{j} \psi_{q}=i \\ 0 & \text { otherwise }\end{cases}
$$

with $M=\sum_{j=1}^{p} M_{j}$. When any output vector is premultiplied by $\Phi$, it extracts the components that correspond to prescribed point-to-point locations, whilst retaining their original order.
Remark 1: If each output is stipulated at the same set of point-to-point locations, that is $\mathcal{S}_{j}=\mathcal{S}_{1}, \forall j \in\{2, \ldots, p\}$, then matrix $\Phi$ has block-wise components

$$
\Phi_{i, j}= \begin{cases}I_{p} & \text { if } j=n_{1, i}, \quad i=1,2, \ldots M_{1} \\ 0_{p} & \text { otherwise. }\end{cases}
$$

In addition the reference has the form

$$
\mathbf{y}_{r}=\left[\mathbf{y}_{r}(0)^{T}, \mathbf{y}_{r}(1)^{T}, \cdots, \mathbf{y}_{r}\left(M_{1}-1\right)^{T}\right]^{T} \quad \in \mathbb{R}^{M}
$$

where $\mathbf{y}_{r}(i) \in \mathbb{R}^{p}$ is the prescribed output vector at sample $n_{1, i}$, and $M=p M_{1}$.

ILC can be formulated for the point-to-point case by deriving an iterative numerical solution to the problem of finding a control input which minimizes the point-to-point error norm. The control objective is to find a sequence of control inputs $\left\{\mathbf{u}_{k}\right\}$ such that

$$
\lim _{k \rightarrow \infty}\left\|\mathbf{y}_{r}-\Phi G \mathbf{u}_{k}\right\|=0
$$

which replaces the standard requirement (2). The ILC update (4) now assumes the form

$$
\mathbf{u}_{k+1}=\mathbf{u}_{k}+L\left(\mathbf{y}_{r}-\Phi \mathbf{y}_{k}\right)
$$

so that the convergence condition (5) becomes

$$
\rho\left(I_{M}-\Phi G L\right)<1
$$

In Section III a learning operator $L$ is derived to satisfy (12), but first further motivation is provided to support the utility of point-to-point ILC over the standard framework.

\section{B. Point-to-point ILC motivation}

Let $d$ denote the rank deficiency of the plant matrix $G$ (the number of linearly dependent rows). If $d>0$ the standard ILC update (4) cannot force the plant to track an arbitrary reference trajectory $\mathbf{y}_{d}$.

Theorem 1: The point-to-point update (11) can enforce tracking of an arbitrary reference $\mathbf{y}_{r}$ if and only if the tracked points are chosen such that

$$
M \leq N p-\max \{d, N(p-m)\}
$$

Proof. A necessary and sufficient condition for an operator $L$ to exist satisfying the convergence condition (12) is that $\operatorname{rank}(\Phi G)=M$. For the standard ILC case $\Phi=I, M=N$ and hence $\operatorname{rank}(\Phi G)=N-d<M$, leading to $I_{M}-\Phi G L$ having $d$ eigenvalues at unity. Now the $i^{\text {th }}$ row of $\Phi G$ is the $\left(j \mid \Phi_{i, j}=1\right)^{t h}$ row of $G$, hence if $p \leq m$ and the pointto-point samples are chosen to correspond to any subset of linearly independent rows of $G$, the convergence condition (12) can be satisfied. If $p>m$ then the additional condition $M \leq N m$ is imposed.

Remark 2: Let system (1) be written as discrete transferfunction matrix $G(z)=C\left(z I_{n}-A\right)^{-1} B+D$ with component $G_{i, j}(z)$ corresponding to the $i^{\text {th }}$ output and $j^{\text {th }}$ input. If the relative degree of $G_{i, j}(z)$ is $r_{i, j}$, then $d=\sum_{i, j} r_{i, j}$.

The ability of point-to-point ILC to employ a modified standard reference to recover feasibility is extremely important, especially as $d \geq 1$ in practice due to the delay action of 
a zero-order hold. However many tasks are naturally defined only at a small number of points, and hence additional benefits may also be expected by not enforcing unnecessary tracking. The next lemma shows how the space of feasible inputs expands as the number of tracked points, $M$, reduces.

Lemma 1: Assuming (13) is satisfied, the feasible input space which forces the system (1) to track $\mathbf{y}_{r}$ is of dimension $p N-M$, and is given by $\mathcal{U}=$ $\left\{(\Phi G)^{\dagger} \mathbf{y}_{r}+\mathbf{x}, \mathbf{x} \in \operatorname{null}(\Phi G)\right\}$ where $A^{\dagger}=\left(A^{T} A\right)^{-1} A^{T}$ is the pseudoinverse of $A$. The nullspace of $\Phi G$ has an orthogonal basis given by the rows of $\overline{\Phi G} P_{(\Phi G)^{T}}^{\perp}$, where $\overline{\Phi G} \in \mathbb{R}^{(p N-M) \times m N}$ is such that the matrix $\left[\overline{\Phi G}^{T}(\Phi G)^{T}\right]$ is full rank, and $P_{A}^{\perp}=I-A A^{\dagger}$ is the orthogonal projection onto the nullspace of $A$.

The algorithm proposed in the next section exploits this enlarged space to achieve desirable performance properties.

\section{GRADIENT DESCENT POINT-TO-POINT ILC}

The gradient descent method is one of many numerical algorithms used to tackle nonlinear optimization problems, and has previously been applied within the standard ILC framework [10]. Motivated by (10) and the accompanying discussion, it is applied to solve

$$
\min _{\mathbf{u}} J(\mathbf{u}), \quad J(\mathbf{u})=\left\|\mathbf{y}_{r}-\Phi G \mathbf{u}\right\|^{2}
$$

leading to the iterative update for the control input

$$
\begin{aligned}
\mathbf{u}_{k+1} & =\mathbf{u}_{k}-\frac{\beta}{2} \nabla_{\mathbf{u}} J\left(\mathbf{u}_{k}\right) \\
& =\mathbf{u}_{k}+\beta(\Phi G)^{T}\left(\mathbf{y}_{r}-\Phi \mathbf{y}_{k}\right)
\end{aligned}
$$

where the experimental plant output, $\mathbf{y}_{k}$ has replaced the nominal value, $G \mathbf{u}_{k}$, so that the optimisation is robustly achieved within the ILC framework.

Theorem 2: Let $\Phi$ comprise point-to-point locations satisfying $\operatorname{rank}(\Phi G)=M$. Let $\tilde{\Phi}$ equal $\Phi$ but with the $i^{\text {th }}$ row removed, and hence correspond to tracking all but the $i^{\text {th }}$ point-to-point location. Let the $M$ eigenvalues of the matrix $A=(\Phi G)(\Phi G)^{T}$ be denoted $\lambda_{M} \leq \lambda_{M-1} \cdots \leq$ $\lambda_{2} \leq \lambda_{1}$, which also equal the singular values since $A$ is Normal. Similarly, let the $M-1$ eigenvalues of the matrix $B=(\tilde{\Phi} G)(\tilde{\Phi} G)^{T}$ be denoted $\mu_{M} \leq \mu_{M-1} \cdots \leq \mu_{3} \leq \mu_{2}$, which also equal the singular values since $B$ is Normal. Then the following relationship holds

$$
\lambda_{M} \leq \mu_{M} \leq \lambda_{M-1} \leq \mu_{M-1} \cdots \leq \mu_{3} \leq \lambda_{2} \leq \mu_{2} \leq \lambda_{1} .
$$

In particular, let $\mathbf{y}$ equal the $i^{\text {th }}$ column of $A$ with the $i^{\text {th }}$ element removed. Then if the eigenvalues of $B$ are distinct and no eigenvector of $B$ is orthogonal to $\mathbf{y}$ then

$$
\lambda_{M}<\mu_{M}<\lambda_{M-1}<\mu_{M-1} \cdots<\mu_{3}<\lambda_{2}<\mu_{2}<\lambda_{1} .
$$

Proof. First note that $A$ is a Hermitian matrix of order $M$, and that $B$ is a principal submatrix of $A$ of order $M-1$. Then (16) follows as an application of Cauchy's Interlace Theorem for eigenvalues of Hermitian matrices [11]. It is further proven in [11] that (17) holds provided: 1) the eigenvalues of $B$ satisfy $\mu_{M}<\mu_{M-1} \cdots<\mu_{3}<\mu_{2}$, and 2) the vector

$$
U^{T} \mathbf{y}=\left[z_{2}, z_{3}, \ldots, z_{M}\right]^{T}
$$

has non-zero elements, where $U$ is a unitary matrix of order $M-1$ such that $U^{T} B U=D$, with $D=$ $\operatorname{diag}\left\{\mu_{2}, \mu_{3} \ldots \mu_{M}\right\}$. To satisfy 2 ) a suitable choice for $U$ has columns that are the eigenvectors of $B$, and hence $\exists z_{i}=0$ only if $\mathbf{y}$ is orthogonal to an eigenvector of $B$.

\section{A. Convergence Rate}

Theorem 3: Provided the point-to-point locations are chosen to satisfy (13), the choice of gain in (15)

$$
0<\beta<\frac{2}{\bar{\sigma}\left(\Phi G(\Phi G)^{T}\right)}=\frac{2}{\left\|\Phi G(\Phi G)^{T}\right\|}
$$

guarantees convergence of the plant output to the reference $\mathbf{y}_{r}$. In particular, the maximum convergence rate corresponds to

$$
\beta=\frac{2}{\bar{\sigma}\left(\Phi G(\Phi G)^{T}\right)+\underline{\sigma}\left(\Phi G(\Phi G)^{T}\right)}
$$

The convergence rate using (20) increases as the number of point locations, $M$, is reduced.

Proof. The convergence condition for (15) corresponds to

$$
\rho\left(I_{M}-\beta \Phi G(\Phi G)^{T}\right)<1
$$

providing a linear convergence rate to zero error [12]. Since $\sigma_{i}\left(\Phi G(\Phi G)^{T}\right)>0, \forall i$ since $\Phi G(\Phi G)^{T}$ is positive definite,

$$
\begin{aligned}
\sigma_{i}\left(I_{M}-\beta \Phi G(\Phi G)^{T}\right) & =1-\beta \sigma_{i}\left(\Phi G(\Phi G)^{T}\right)<1 \quad \forall i \\
& \Rightarrow 0<\beta \sigma_{i}\left(\Phi G(\Phi G)^{T}\right)<2
\end{aligned}
$$

yielding (19). The solution to $\min _{\beta} \rho\left(I-\beta \Phi G(\Phi G)^{T}\right)$ corresponds to the choice (20) and convergence rate

$$
\begin{aligned}
& \frac{\bar{\sigma}\left(\Phi G(\Phi G)^{T}\right)+\underline{\sigma}\left(\Phi G(\Phi G)^{T}\right)}{\bar{\sigma}\left(\Phi G(\Phi G)^{T}\right)-\underline{\sigma}\left(\Phi G(\Phi G)^{T}\right)}= \\
& 1+\frac{2 \underline{\sigma}\left(\Phi G(\Phi G)^{T}\right)}{\bar{\sigma}\left(\Phi G(\Phi G)^{T}\right)-\underline{\sigma}\left(\Phi G(\Phi G)^{T}\right)}
\end{aligned}
$$

Application of Theorem 2 guarantees that each point removed from $\mathbf{y}_{r}$ increases $\underline{\sigma}\left(\Phi G(\Phi G)^{T}\right)$ and reduces $\bar{\sigma}\left(\Phi G(\Phi G)^{T}\right)$. Hence the convergence rate increases.

The convergence can be further analysed by diagonalizing the matrix relationship $\Phi e_{k+1}=\left(I-\beta \Phi G(\Phi G)^{T}\right) \Phi e_{k}$

$$
\Phi e_{k+1}=V \operatorname{diag}\left\{1-\beta \sigma_{i}\left(\Phi G(\Phi G)^{T}\right)\right\} V^{-1} \Phi e_{k}
$$

where $V$ is the eigenvector matrix of $\Phi G(\Phi G)^{T}$. This gives

$$
V^{-1} \Phi e_{k}=\operatorname{diag}\left\{\left(1-\beta \sigma_{i}\left(\Phi G(\Phi G)^{T}\right)^{k}\right\} V^{-1} \Phi e_{0} .\right.
$$

Since $V^{-1}=V^{T}$, the component of $\Phi e_{0}$ projected onto the $j^{\text {th }}$ eigenvector of $\Phi G(\Phi G)^{T}$ hence evolves as $(1-$ $\left.\beta \sigma_{i}\left(\Phi G(\Phi G)^{T}\right)\right)^{K}$. If eigenvalues $i_{a}$ and $i_{b}$ correspond to minimum and maximum values of $\left|1-\beta \sigma_{i}\left(\Phi G(\Phi G)^{T}\right)\right|$, then the point-to-point reference with the fastest convergence rate is given by a scalar multiple of $\mathbf{y}_{r}=V_{i_{a}}$, and similarly the slowest is $\mathbf{y}_{r}=V_{i_{b}}$. For an arbitrary reference, the error norm sequence lies in the interval

$\left(1-\beta \sigma_{i_{a}}\left(\Phi G(\Phi G)^{T}\right)\right)^{k} \leq \frac{\left\|\Phi e_{k}\right\|}{\left\|\Phi e_{o}\right\|} \leq\left(1-\beta \sigma_{i_{b}}\left(\Phi G(\Phi G)^{T}\right)\right)^{k}$ 


\section{B. Robustness Margins}

Theorem 4: Let there exist a multiplicative uncertainty on each element of the plant model $G(z)$, such that $\check{G}_{i, j}(z)=$ $G_{i, j}(z) U_{i}(z)$. Here $\check{G}(z)$ is the actual plant and the model $G(z)$ corresponds to the matrix $G$ used in the update law (15). A sufficient condition for monotonic convergence is that each $\arg \left\{U_{i}\left(e^{j \omega}\right)\right\}$ lies in the open interval $(-\pi / 2, \pi / 2)$, demonstrating a phase margin of $90^{\circ}$.

Proof. This is an extension of robustness analysis for the standard gradient algorithm $\left(\Phi=I_{p N}\right)$ in [13] for the SISO case. Suppose that the uncertainty can be expressed in the matrix form $\breve{G}=G U$, and that point locations satisfy (13). Then from (15) the point-to-point error satisfies

$$
\begin{aligned}
\left\|\tilde{e}_{k+1}\right\|^{2}-\left\|\tilde{e}_{k}\right\|^{2}= & \beta^{2} \tilde{e}_{k}^{T} \Phi G \check{G}^{T} \Phi^{T}\left(\Phi G \check{G}^{T} \Phi^{T}\right)^{T} \tilde{e}_{k} \\
& -\beta \tilde{e}_{k}^{T} \Phi G U^{T} G^{T} \Phi^{T} \tilde{e}_{k}
\end{aligned}
$$

where $\tilde{e}=\Phi e$. If $U$ is positive, the first term on the righthand side is strictly positive for an arbitrary non-zero $\tilde{e}_{k}$ and $\beta>0$, and of $\mathcal{O}\left(\beta^{2}\right)$. Similarly the second term is of $\mathcal{O}(\beta)$ and strictly negative, and hence there always exists a $\beta>0$ which ensures monotonic reduction in error norm. This also holds if the components of $\tilde{e}_{k}^{T} \Phi G$ are reordered so that the elements corresponding to the same input are grouped, resulting in a reordering of the $U$ matrix such that $U_{(i-1) m+i,(j-1) m+j}=U_{i, j}$. The stipulation that the components of $G$ associated with the same input have the same uncertainty then results in $U$ having the block diagonal structure $U=\left\{U_{1}, U_{2} \ldots U_{m}\right\}$ where $U_{i}$ corresponds to the $i^{t h}$ input. A sufficient condition for $U$ to be positive definite is that each $U_{i}$ is positive definite. This is the same as that arrived in [13] which goes on to show that a sufficient condition is that each $U_{i}(z)$ is positive-real. Therefore a sufficient condition for monotonic convergence is that $\arg \left\{U_{i}\left(e^{j \omega}\right)\right\}$ lies in the open interval $(-\pi / 2, \pi / 2)$ $\forall i$. Note that any gain uncertainty $\left|U_{i}\left(e^{j \omega}\right)\right|$ can be tolerated through use of a sufficiently small $\beta$.

\section{Input Energy}

Theorem 5: Consider the system (1) and a point-to-point reference $\mathbf{y}_{r}$. ILC algorithm (15) converges to the minimum input energy solution that tracks $\mathbf{y}_{r}$. Furthermore, this solution is bounded by

$$
\|\mathbf{u}\| \leq \frac{\left\|\mathbf{y}_{r}\right\|}{\underline{\sigma}\left(\Phi G(\Phi G)^{T}\right)}
$$

whose right-hand side strictly reduces as the number of points $M$ is reduced.

Proof. Repeated application of (15), with $\mathbf{u}_{0}=\mathbf{0}$, yields

$$
\mathbf{u}_{k+1}=\left(\sum_{i=0}^{k}\left(I-(\Phi G)^{T} \Phi G\right)^{i}\right)(\Phi G)^{T} \mathbf{y}_{r}
$$

which has the limit

$$
\lim _{k \rightarrow \infty} \mathbf{u}_{k}=\left((\Phi G)^{T} \Phi G\right)^{-1}(\Phi G)^{T} \mathbf{y}_{r}=(\Phi G)^{\dagger} \mathbf{y}_{r}
$$

Now suppose the input update $\mathbf{u}^{*}$ solves the standard tracking problem, so that $G \mathbf{u}^{*}=\mathbf{y}_{d}$ where $\mathbf{y}_{d}$ contains the desired points, $\mathbf{y}_{r}$. Then exchange rows in matrix $G$ and $\mathbf{y}_{d}$ to group the stipulated, $\mathbf{y}_{r}$, and free components, $\overline{\mathbf{y}}_{d}$, as

$$
\left[\begin{array}{c}
\bar{\Phi} G \\
\Phi G
\end{array}\right] \mathbf{u}^{*}=\left[\begin{array}{l}
\overline{\mathbf{y}}_{d} \\
\mathbf{y}_{r}
\end{array}\right]
$$

where $\bar{\Phi} \in \mathbb{R}^{(p N-M) \times p N}$ is such that $\left[\bar{\Phi}^{T}, \Phi^{T}\right]$ is full rank. The optimal cost of the problem

$$
\min _{\mathbf{u}}\|\mathbf{u}\| \text { subject to } \Phi G \mathbf{u}=\mathbf{y}_{r}
$$

is the norm of the orthogonal projection of $\mathbf{u}^{*}$, onto the range of $(\Phi G)^{T}$, that is

$$
\|\mathbf{u}\|=\left\|(\Phi G)^{T}\left((\Phi G)^{T}\right)^{\dagger} \mathbf{u}^{*}\right\| \leq\left\|\mathbf{u}^{*}\right\|
$$

Now insert into (26) the relationship

$$
\mathbf{u}^{*}=\left[\left(\bar{\Phi} G P_{(\Phi G)^{T}}^{\perp}\right)^{\dagger},\left(\Phi G P_{(\bar{\Phi} G)^{T}}^{\perp}\right)^{\dagger}\right]\left[\begin{array}{l}
\overline{\mathbf{y}}_{d} \\
\mathbf{y}_{r}
\end{array}\right]
$$

to obtain a value of $\mathbf{u}$ which equates to the limit given in (24). The relationship

$$
\left\|(\Phi G)^{\dagger}\right\|=\bar{\sigma}\left((\Phi G)^{\dagger}\right)=\frac{1}{\underline{\sigma}\left(\Phi G(\Phi G)^{T}\right)}
$$

where $\bar{\sigma}$ and $\underline{\sigma}$ are the maximum and minimum singular values respectively, leads to (23). It follows that the input norm is small when point-to-point locations are selected which maximize the smallest eigenvalue of $\Phi G(\Phi G)^{T}$. Applying Theorem 2 means that $\underline{\sigma}\left(\Phi G(\Phi G)^{T}\right)$ increases as each point-to-point location is removed, and hence the righthand side of (23) reduces.

Remark 3: If only a single point is specified for each output then (23) becomes

$$
\|\mathbf{u}\| \leq \frac{\left\|\mathbf{y}_{r}\right\|}{\min _{j=1, \ldots, p} \sum_{q=1}^{m}\left\|G_{j, q}(z)\right\|}
$$

This is also achieved if the time between point locations exceeds the time taken for the impulse response to approximately go to zero (assuming asymptotic stability).

Theorem 5 provides an example of the benefit obtained compared with the bound $\|\mathbf{u}\|=\left\|G^{-1} \mathbf{y}_{r}\right\|$ corresponding to standard ILC (if it exists). This benefit increases as the number of tracked points is reduced, or their temporal spacing is increased.

\section{Practical Implementation}

Remark 4: The term $(\Phi G)^{T}\left(\mathbf{y}_{r}-\Phi \mathbf{y}_{k}\right)$ in (15) can be efficiently generated using the co-state representation of system (1). More specifically, it is equal to the output $\tilde{\mathbf{y}}$ of the system

$$
\begin{aligned}
\tilde{\mathbf{x}}(t) & =A^{T}(t+1) \tilde{\mathbf{x}}(t+1)+C^{T}(t+1) \tilde{\mathbf{u}}(t+1) \\
\tilde{\mathbf{y}}(t) & =B^{T}(t) \tilde{\mathbf{x}}(t)+D^{T}(t) \tilde{\mathbf{u}}(t), \quad t=N-1, \ldots, 0
\end{aligned}
$$

with the input $\tilde{\mathbf{u}}=\Phi^{T}\left(\mathbf{y}_{r}-\Phi \mathbf{y}_{k}\right)$. Use of the co-state system therefore avoids calculation of the large $G$ matrix appearing in (15) and the algorithms which follow. 


\section{MULTIVARIABLE TEST FACILITY}

The performance advantages established in the previous section are now confirmed experimentally using a multivariable test facility. This system employs two interconnected differential gearboxes, together with spring-mass-damper components and is driven by two induction motors [14]. A high degree of coupling between inputs and outputs is possible which can be varied by the operator. The completed system is shown in Fig. 1 with component details listed in Table I. The level of damping applied between the two differential gearboxes, shown as component 11 in Table I, is parametrized by $c \in[0,1]$ which determines the interaction existing in the system. Modeling of each interaction level has been achieved through open-loop frequency response tests, in which sinusoids were injected over the range of interest. The resulting output power spectra were used to generate Bode plots which have been optimally fitted by linear transferfunctions. For interaction level $c$ the resulting model is

$$
\left[\begin{array}{l}
\phi_{1} \\
\phi_{2}
\end{array}\right]=\left[\begin{array}{ll}
H_{B 1}^{c} & H_{B 2}^{c} \\
H_{C 1}^{c} & H_{C 2}^{c}
\end{array}\right]\left[\begin{array}{l}
U_{1} \\
U_{2}
\end{array}\right]
$$

where $\phi_{1}$ and $\phi_{2}$ are outputs (rad) of the MIMO system that are measured by encoders, $U_{1}, U_{2}$ are the inputs, (volts), fed to the inverters. For the uncoupling case $(c=0)$ the transferfunction components are

$$
\begin{array}{lc}
H_{B 1}^{0}=\frac{130000(s+2.75)}{s(s+12.58)(s+14.20)(s+13.08)(s+15.10)} \\
H_{C 2}^{0}=\frac{50000(s+1.99)}{s(s+8.41)(s+9.53)(s+10.62)(s+12.60)} \\
H_{B 2}^{0}= & 0 \\
H_{C 1}^{0}= & 0
\end{array}
$$

and for the fully coupling case $(c=1)$

$$
\begin{aligned}
& H_{B 1}^{1}=\frac{1403008200(s+0.06)}{s(s+45.0)(s+43.4)(s+38.2)(s+25.4)(s+31.7)(s+29.2)} \\
& H_{B 2}^{1}=\frac{5000000(s+0.77)}{s(s+30.09)(s+28.43)(s+38.91)^{2}(s+40.66)} \\
& H_{C 1}^{1}=\quad \frac{59008000(s+1.89)}{s(s+38.07)(s+47.25)(s+49.32)(s+37} \\
& H_{C 2}^{1}=\frac{s(s+38.07)(s+47.25)(s+49.32)(s+37.80)(s+28.19)}{s(s+36.20)(s+39.65)(s+59.72)(s+39.04)(s+38.41)(s+60.93)}
\end{aligned}
$$

For other values of $c$ transfer-functions were similarly obtained. A proportional feedback loop has been implemented as a prestabilizer around each transfer-function, using a gain of 0.1 . The closed-loop system constitutes the controlled system and, following discretization using $T_{s}=0.01$, is converted to matrix $G$ using (3). $T=5$ secs giving $N=500$.

First standard ILC is applied using $M=N, \Phi=I$, in the update (15) with optimal $\beta$ calculated from (20). Sinusoidal references are used, given by $\mathbf{y}_{r}=\left[r_{1}(0) r_{2}(0) \ldots r_{1}(N-\right.$ 1) $\left.r_{2}(N-1)\right]^{T}$ where $r_{1}(t)=6 \sin (2 \pi t / T), r_{2}(t)=$ $3 \sin (2 \pi t / T)$. The control input and error norms are shown in Fig. 2 and Fig. 3 respectively. A very high input is required which increases with the coupling level, attaining a value of $\approx 1700$. This would continue to increase but due to safety reasons the experiment was terminated after 1200 trials, and the coupling level was limited to 0.9. The error convergence is very slow, and is still high after 1200 trials with the coupling level $c=0.9$. The predicted convergence rate is

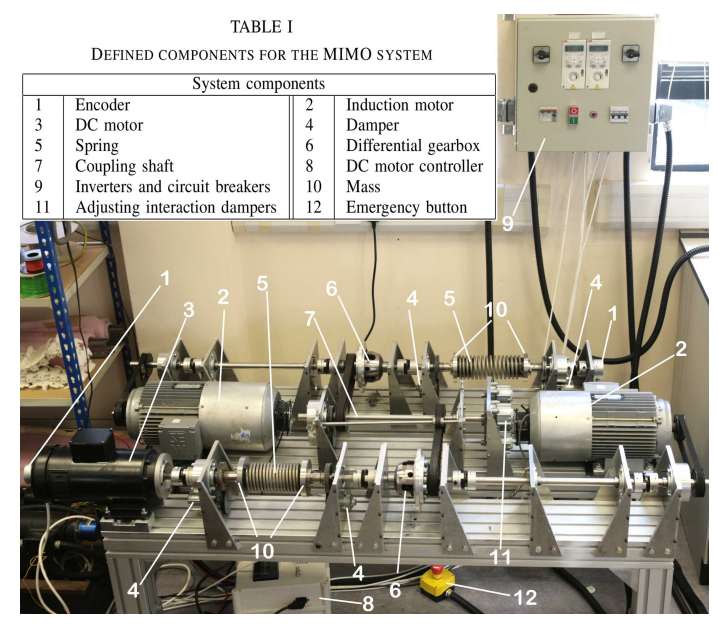

Fig. 1: MIMO testbed facility
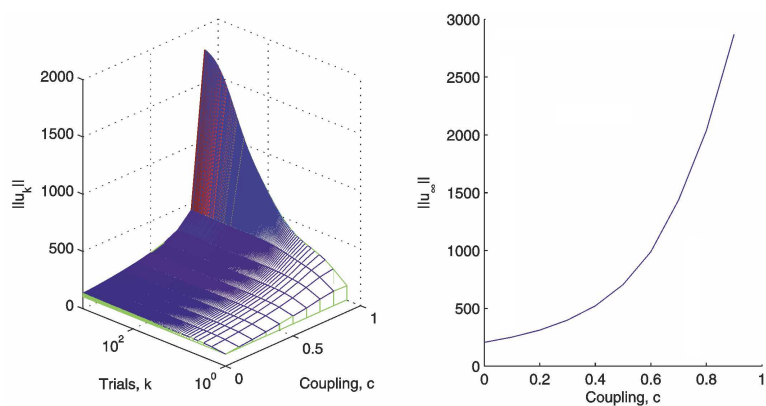

Fig. 2: Standard ILC input norm: a) Experimental results, and b) Predicted final value.

calculated using (22) and differs from the results due to model uncertainty and noise that are exacerbated by the large control inputs and high number of trials.

Ten points have been selected from the references $r_{1}(t)$, $r_{2}(t)$ for use with point-to-point ILC. These are respectively $\mathbf{y}_{r, 1}=[3.53,5.71,0,-5.71,-3.53]$ at time points $\mathcal{S}_{1}=\{50$, $150,250,350,450\}$ and $\mathbf{y}_{r, 2}=[2.85,1.76,-1.76,-2.85$, $0]$ at time points $\mathcal{S}_{2}=\{100,200,300,400,500\}$. The reference $\mathbf{y}_{r}$ is assembled according to Section II, together with the $10 \times N$ matrix $\Phi$. The results are shown in Fig. 4 and Fig. 5. The convergence is far faster than the standard case, and the input norm far reduced. The predicted convergence rate (22) is also shown, and matches the experimental data more accurately than for standard ILC. The standard ILC framework is again applied, but the sinusoidal references are now replaced with the reference

$$
\mathbf{y}_{d}=G(\Phi G)^{\dagger} \mathbf{y}_{r}
$$

which solves (14) based on the nominal plant model. This ensures fair comparison with point-to-point ILC. Results are shown in Fig. 6 and Fig. 7. Both point-to-point and standard ILC with reference (29) show that a higher coupling level reduces convergence speed and requires greater control effort. Comparing the error norms, the results show that point-to-point ILC achieves a lower error norm, and this is accentuated as the level of coupling is increased. This difference is due to the standard ILC framework enforcing tracking of unnecessary points which gives rise to learning 

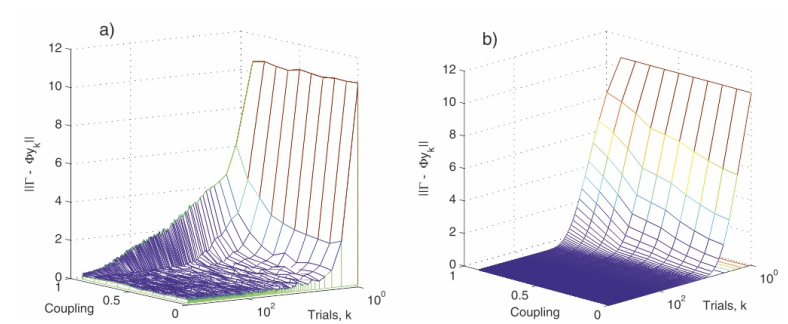

Fig. 3: Standard ILC error norm: a) Experimental and b) Predicted results.
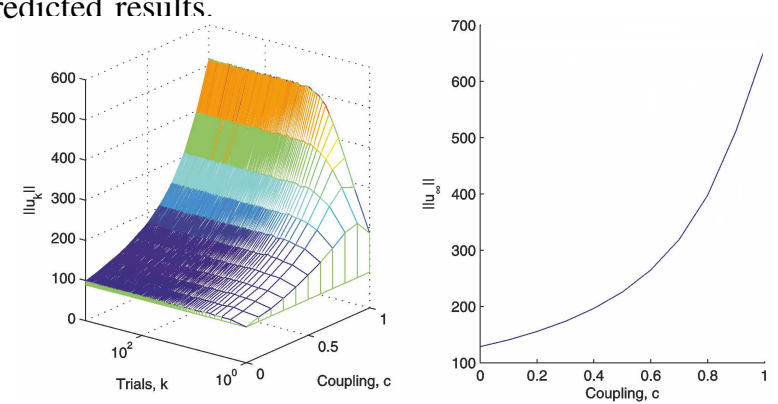

Fig. 4: Point-to-point ILC input norm: a) Experimental results, and b) Predicted final value.

transients which are magnified by noise and plant model uncertainty. The predicted convergence rate is calculated using (22) and is also shown. This is accurate for approximately the first 100 trials but the presence of model uncertainty and noise causes discrepancies thereafter. For coupling levels [0.1 $\begin{array}{lllllllll}0.2 & 0.3 & 0.4 & 0.5 & 0.6 & 0.7 & 0.8 & 0.9 & 1]\end{array}$ the predicted convergence rates for point-to-point ILC are [2.31, 2.26, 2.165, 2.06, 1.96, $1.92,1.85,1.71,1.50,1.37,1.29]$ and for standard ILC with reference $(29)$ it is $[1.83,1.79,1.71,1.63,1.54,1.50,1.47$, $1.35,1.27,1.22,1.16]$. This increased rate reflects Theorem 3.

The results show that point-to-point ILC provides superior convergence and a reduced input norm for controlling the MIMO testbed facility, especially at high interaction levels.

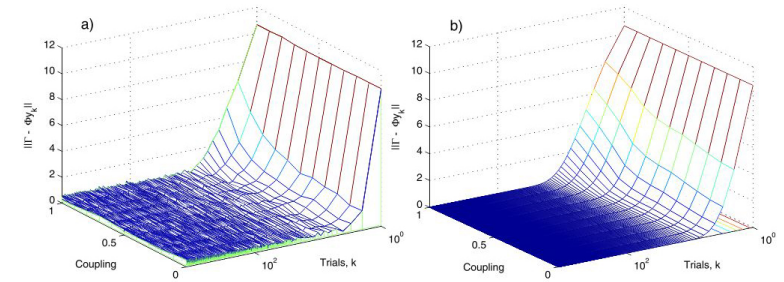

Fig. 5: Point-to-point ILC error norm: a) Experimental and b) Predicted results.
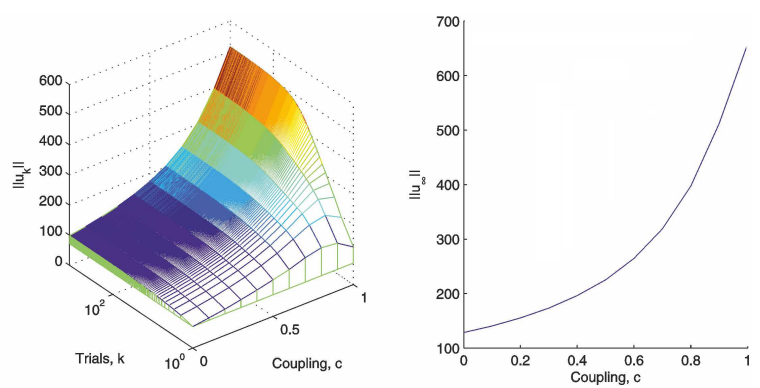

Fig. 6: Standard ILC with optimal $y_{d}$ input norm: a) Experimental results, and b) Predicted final value.

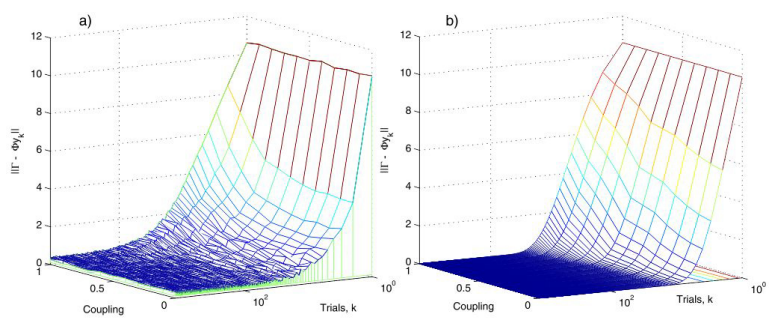

Fig. 7: Standard ILC with optimal $y_{d}$ error norm: a) Experimental and b) Predicted results.

\section{CONCLUSIONS}

The requirement for point-to-point motion control arises in many practical applications, including industrial automation, robotics and rehabilitation engineering. However, there are no available approaches to address general point-to-point tasks and given performance objectives in a framework which uses learning to attain optimal solutions in the presence of model uncertainty and noise. This paper derives an algorithms to address this deficit, and analyses convergence, performance and robustness properties. Experimental results confirm the practical utility and performance of the proposed approaches and illustrate the benefit gained over using the standard framework with an a priori generated reference.

\section{REFERENCES}

[1] H. S. Ahn, Y. Chen, and K. L. Moore, Iterative learning control: Brief survey and categorization, IEEE Transactions on Systems, Man, and Cybernetics, Part C, vol. 37, no. 6, pp. 1099-1121, 2007.

[2] H. Ding and J. Wu, Point-to-point control for a high-acceleration positioning table via cascaded learning schemes,IEEE Transactions on Industrial Electronics, vol. 54, no. 5, pp. 2735-2744, 2007.

[3] J. Park, P. H. Chang, H. S. Park, and E. Lee, Design of learning input shaping technique for residual vibration suppression in an industrial robot, IEEE/ASME Transactions on Mechatronics, vol. 11, no. 1, pp. $55-65,2006$.

[4] J. van de Wijdeven and O. Bosgra, Residual vibration suppression using hankel iterative learning control, Internation Journal of Robust and Nonlinear Control, vol. 18, pp. 1034-1051, 2008.

[5] G. Gauthier and B. Boulet, Robust design of terminal ILC with $H_{\infty}$ mixed sensitivity approach for a thermoforming oven, Journal of Manufacturing Science and Engineering, p. Article ID 289391, 2008.

[6] Y. Wang and Z. Hou, Terminal iterative learning control based station stop control of a train, International Journal of Control, vol. In Press, 2011.

[7] J.-X. Xu and D. Huang, Initial state iterative learning for final state control in motion systems, Automatica, vol. 44, pp. 3162-3169, 2008.

[8] J.-X. Xu, Y. Chen, T. Lee, and S. Yamamoto, Terminal iterative learning control with an application to RTPCVD thickness control, Automatica, vol. 35, pp. 1535-1542, 1999.

[9] P. Lucibello, S. Panzieri, and G. Ulivib, Repositioning control of a twolink flexible arm by learning, Automatica, vol. 33, no. 4, pp. 579-590, 1997.

[10] D. H. Owens, J. J. Hätönen, and S. Daley, Robust monotone gradientbased discrete-time iterative learning control, International Journal of Robust and Nonlinear Control, vol. 19, pp. 634-661, 2009.

[11] S.-G. Hwang, Cauchy's interlace theorem for eigenvalues of Hermitian matrices, The American Mathematics Monthly, vol. 111, pp. 157-159, 2004.

[12] J. M. Ortega and W. C. Rheinboldt, Iterative Solution of Nonlinear Equations in Several Variables, London: Academic Press, 1970.

[13] J. J. Hätönen, Issues of algebra and optimality in iterative learning control, Ph.D. dissertation, Department of Process and Environmental Engineering, University of Oulu, 2004.

[14] T. V. Dinh, C. T. Freeman, and P. L. Lewin, Development of a multivariable test facility for the evaluation of iterative learning controllers, American Control Conference, Montreal, 2012, to be published. 